\title{
Design on Fire Alarm System Based on BP Neural Network and Multiple Source Information Fusion
}

\author{
Wei Wang \\ College of Computer Science and Technology \\ Changchun University \\ Changchun, China \\ e-mail: wangweitea@163.com \\ Li-ping Yang \\ College of Computer Science and Technology \\ Changchun University \\ Changchun, China \\ e-mail: yanglipingtea@163.com
}

\author{
Bo Jiang \\ College of Optical and Electronical Information \\ Changchun University of Science and Technology \\ Changchun, China \\ e-mail: 351649779@qq.com
}

\begin{abstract}
The infrared and smoke sensors widely applied in fire alarm and fire monitoring in the scene of large indoor and outdoor warehouse is vulnerable to sorts of interference noise, it may out of work to cause error fire alarm or fire omission. Based on design of reliable flame recognition algorithm, a practical intelligent fire alarm system based on BP neural network and multiple source information was constructed. The system uses multi-channel time-division multiplexing technology to constitute temperature detector, photoelectric smoke detector and infrared CCD detector into multiple source detector set. The information fusion and BP neural network were used for accurate prediction of fire level, which improves reliability of fire alarm and decrease false alarm rate.
\end{abstract}

Keywords- multiple source information fusion; BP neural network; fire alarm system; intelligent

\section{INTRODUCTION}

The high hazard cause by fire is well-known. Most of existing various fire alarm and consumer monitoring devices are based on infrared sensor and some sensors. In the large space scene of outdoor warehouse the large indoor warehouse, the above sensor signal becomes very weak due to huge space. Even the sensors with high accuracy may not work caused by various interference and noise [1-3]. To solve such problem in occasions of fire alarm, some foreign companies carry out the ultraviolet image sensor with a long-range detection of flames in the early 1990s and introduced the corresponding products. But such a system does not have automatic identification function. The module does not have to be reconfigured. The flame of simple identification method is higher recognition rate of false positives. A large number of experimental results show that the combustion in the fire, flame-infrared whose wavelengths greater than $950 \mathrm{~mm}$ is not obviously visible radiation identifiable characteristic, if the radiation collected by the sensor signal, a video signal by the photoelectric conversion characteristics can be identified. Due to large area of the video signal may be monitoring, it can satisfy the requirements of fire monitoring in large space occasions.

The paper uses precision infrared CCD image sensor flame fire scene capture infrared radiation and converts it to a standard black and white video signal. After the sequence image acquisition device, the video signal after the $\mathrm{A} / \mathrm{D}$ converting the digital gray scale image sequence is formed. Finally, the flame image sequences identification method is used to identify in real-time approach to identify fire monitoring. As a result of the band $950-2000 \mathrm{~mm}$ infrared CCD sensor, only the infrared radiation in the band of the video signal is formed, when the flame of infrared radiation is focused on the above wavelength range, the wavelength of the interference signal from another is greatly attenuated [2]. Therefore, when there is a flame burning, the video signal by the $\mathrm{CCD}$, in addition to the formation of high-brightness signal caused by intense flame radiation, but only a small amount of infrared jamming signal, and these signals are often expressed as a fixed image mode, you can use a simple algorithms to classify, so greatly improves the recognition rate of the flame, and the amount of data processing to improve the speed of detection and identification.

On the basis of reliable flame recognition algorithm, the paper constructs a practical intelligent fire alarm system based BP neural network and multi-source information fusion. In order to improve the detection rate of the system, time-division multiplexing multi-channel in this system, the temperature detectors, infrared CCD image detectors and photoelectric smoke detectors constitute multiple source fire detector set, so that same processor can sharing deal with multi-channel monitoring signals. 
Using multi-source information fusion decision methods, it can achieve accurate prediction of fire level to achieve the building fire alarm tasks, improving the reliability of fire alarm and reduce the false alarm rate.

\section{SYSTEM HARDWARE DESIGN}

\section{A. System Architecture}

The system completes functions of monitoring process control, channel image intelligent recognition and humanmachine interaction with microprocessor. In the software system, it uses menu-driven and time-processing technology to allow human-machine interaction at the same time without affecting the real-time monitoring of each channel. The whole system is shown in Figure 1. Intelligent fire alarm detectors are installed in all parts of the field, which are connected through the CAN bus and zone controllers. Intelligent fire detector detects the signal sent to the fire zone controller via CAN bus. After analysis and processing area controller, audible alarm signal and displays the location of the sound and light alarms; while regional control will be collected from each of the fire sensor signal via RS-485 communication interface sent to a centralized controller. Centralized controller is consisted by the IPC to responsible for managing various district systems and achieving functions of alarming printing and others.

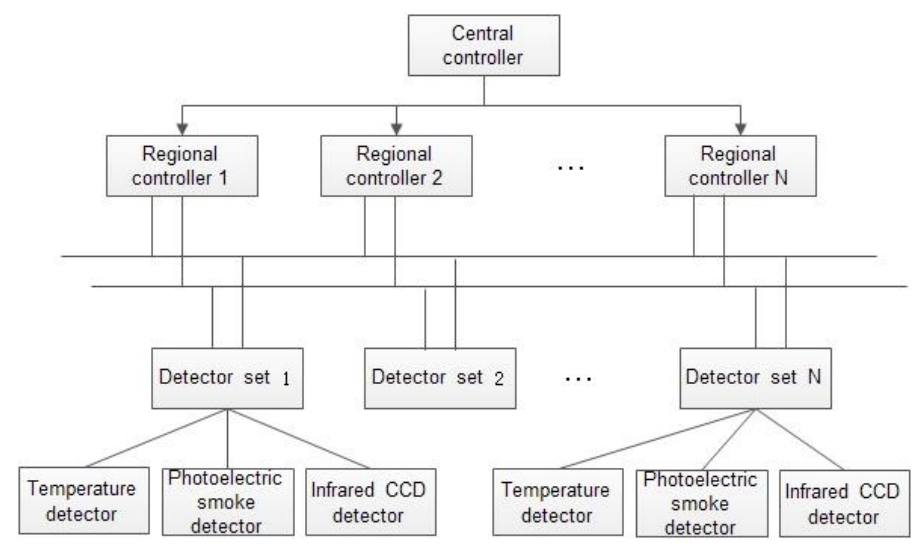

Figure 1. System architecture.

\section{B. Design on Fire Detector}

The core of fire detectors is P89C668 microcontroller from Philips Company. The P8C668 is microcontroller based on 80C51, which has 60kb flash memory. The memory can be either parallel programming or upgrade directly through ISP user, so as to provide a convenient upgrade program. The detector integrates temperature detector and photoelectric smoke detectors to make up for shortcomings of imply using smoke detectors or heat detectors $[4,5]$. As the detector has microcontroller. It can implement complex fuzzy logic reasoning algorithm independently to carry out mathematic analysis, digital filtering and compensation operations. The detectors communicate with central controller with $\mathrm{CAN}$ bus to implement peer communication. It also has characteristics of long-distance communication. The structural principle of intelligent fire detector is shown in Fig. 2.

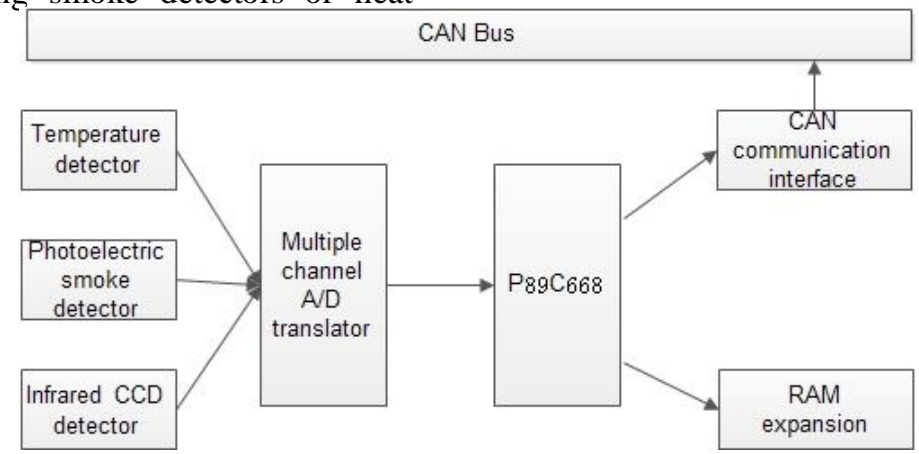

Figure 2. Structure of intelligent fire detector.

\section{Design of Regional/Central Alarm Controller}

Regional alarm controller connects to each detector with CAN bus to complete cycle detection of each detector. If a sensor fails, it will prompt the operator to carry out maintenance, and according to the information sent by the detector, using fuzzy neural network algorithm to analyze all kinds of information, judgment, and make the appropriate treatment based on the result [6-8]. The system provides an input/output machine interface, easy operation personnel to operate the system and obtain system information. Even centralized alarm controller connected via RS-485, and each area alarm controller. On the one hand, and exchange information with each area alarm controller via RS-485 communication lines. On the other hand, it completes a variety of information storage, display, print and other functions. When a fire occurs is determined by an emergency radio, lights, alarm bells, etc., and can be 
based on pre-designed linkage relationships, according to a fire situation, the regional linkage fire alarm controller startup device, automatic fire.

\section{System AlgORITHM DESIGN}

\section{A. Fire Alarm Algorithm Based on BP and Information Fusion}

According to multiple source information fusion theory, it can generate input/output action table for alarm system. Its inputs are smoke concentration characteristic, temperature signal characteristic and infrared image spectral characteristic. Carry out fusion judgment processing on quantized values corresponding to these characteristic values and output fire prediction level. The temperature sensors, smoke sensors and CCD detector detects infrared signals are collected through monitoring system and then digitized for filtering, which acts as input of fire prediction model. According to fusion decision expert system, the corresponding fire prediction level can be obtained. The fire automatic detection algorithm based on NP neural network and multiple source information fusion will greatly improve system intelligence. The sensitivity of fire detection can be effectively improved and system false alarm rate can be reduced. The process is shown in Fig. 3.

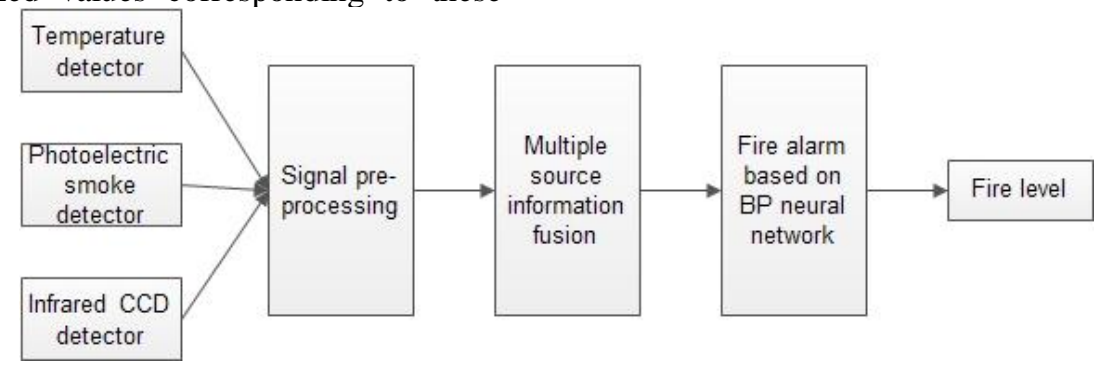

Figure 3. Fire alarm algorithm based on information fusion and BP network.

\section{B. Infrared Image Recognition Algorithm}

Infrared image sequences of the flame has the following characteristics: the adjacent edges of the frame image is unstable; adjacent frames with stable similarity; similarity between images within a certain variation range; flame in the flame of the core part of the image gray greater than the other parts of the gray flame. In the infrared attenuated video signal, the interference pattern signal is mainly the performance of fixed bright and fast moving large infrared illumination changes. Therefore, in recognition of the flame, the interference pattern may be first non-interfering with the flame pattern classification, and then in the non-interfering characteristics of the image pattern recognition of a flame to the flame is determined [9, $10]$.

Assume the digital sequence image is $\left\{f_{i}(x, y)\right\}, i=1, \cdots, N$. The $(x, y)$ is coordinate of each pixel in the image. The $N$ is frame number in image sequence. In the practical system, $N$ is usually larger than 2 . As to each frame digital image $(x, y) \in \Omega, \Omega$ us the area of the whole image. As tp each channel of image signal, the stable based image is set as $\left\{f_{0}(x, y)\right\}$. The system uses base image to represent normal situation of this channel. As to each frame image in image sequence, there is $\delta_{i}(x, y)=\left|\left\{f_{i}(x, y)\right\}-\left\{f_{0}(x, y)\right\}\right|, i=1, \cdots, N,(x, y) \in \Omega$. The $\left\{\delta_{i}(x, y)\right\}$ us a difference sequence, which means between original image sequence $\left\{f_{i}(x, y)\right\}$ and basis $\left\{f_{0}(x, y)\right\}$. The original mode is discriminated on $\left\{\delta_{i}(x, y)\right\}$.

Firstly, carry out binarization on $\left\{\delta_{i}(x, y)\right\}$ to segment transform region of $\left\{\delta_{i}(x, y)\right\}$. When considering the general binarization infrared illumination range of flame, a flame in the high gray value, and has a complex full halftone infrared reflective region. We use the following methods: statistical histogram $P H S(r)$, of $\left\{\delta_{i}(x, y)\right\}$ with a small window on the $P H S(r)$ is smoothed, smoothing the $P H S(r)$ on the scan from high to low, can be detected in the first trough set binarization threshold.

Make binarization on $\left\{\delta_{i}(x, y)\right\}$ with this threshold and obtain $\left\{b_{i}(x, y)\right\}$. The pixel 1 represents region where the original image sequence $\left\{f_{i}(x, y)\right\}$ and base image $\left\{f_{0}(x, y)\right\}$ has significant difference. We believe that the region is likely to flame zone. In some unpredictable factors, it is introduced $\left\{b_{i}(x, y)\right\}$ by the isolated points in the actual system. These points can be isolated using morphological filtering expansion and erosion operation, so that the area to get a better description. However, such amount of calculation cannot be performed in real time. This article uses the domain after four-neighbor traversal algorithm on $\left\{b_{i}(x, y)\right\}$ to be neighbors domain traversal, filter out the effects of isolated points and arrive at $\left\{\hat{b}_{i}(x, y)\right\}$. For a pixel of marking $\left\{\widehat{b}_{i}(x, y)\right\}$, each frame in the image sequence obtained may be flame area $\Omega, i=1, \cdots, N$.

Pre-processing. Set $S_{i}$ as area of $\Omega$. There is following conclusions to value range of $S_{i}$ :

(a) If $S_{i} \leq$ threshold 1 , the changing area are noise points.

(b) If $S_{i} \geq$ threshold 2 , this changing area are infrared light change with large area.

(c) If threshold $1 \leq S_{i} \leq$ threshold 2 , the changing area is suspicious flame region.

Mode classification: After suspicious area been found, the similarity of adjacent frames of changing images can be simply classified on frame and interference pattern. The similarity of adjacent frame changing image is defined as: 


$$
\zeta_{i}=\frac{\sum_{(x, y) \in \Omega} \widehat{b}_{i}(x, y) \cap \widehat{b}_{i+1}(x, y)}{\sum_{(x, y) \in \Omega} \widehat{b}_{i}(x, y) \bigcup \widehat{b}_{i+1}(x, y)} ; i=1, \cdots, N-1
$$

As to each $\zeta_{i}$, its average value is $\zeta=\frac{1}{N-1} \sum_{i=1}^{N-1} \zeta_{i}$. There is following determination rules:

(a) If $\zeta \leq$ threshold 1 , the image pattern is rapid moving highlight.

(b) If $\zeta \geq$ threshold 2 , the image pattern is fixed infrared light emitting region.

(c) If threshold $1 \leq \zeta \leq$ threshold 2 , the image pattern is flame.

\section{SOFTWARE DESIGN}

The software in system central controller is implemented with object-oriented programming languageDelphi. The central controller constantly finds and process data from each regional controller. Once the fire signal been found, it initiates display, print and alarm light.

The software design on system regional controller includes main program, system initialization subprogram, keyboard scan subprogram, fire processing subprogram and fault processing subprogram. The detector software mainly completes extraction and sampling of each sensor signal. The fuzzy neural network algorithm is used to complete communication with CAN bus. The software flow is shown in Fig. 4.

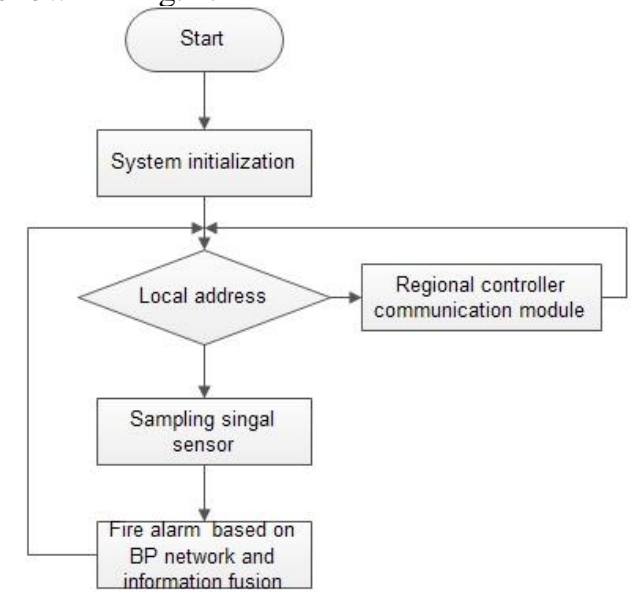

Figure 4. Fire alarm algorithm based on information fusion and BP network.

\section{CONCLUSION}

The system make full use of features that multiple information fusion to express human experiences and deal with fuzzy information. Combining with functions of selflearning and self-adjustment of BP neural network, the intelligent fire alarm system based on fuzzy neural network was constructed. The system detector integrates high-performance microprocessor to collection processing of various sensors. The transmission bus uses CAN bus to enhance communication reliability and prolong transmission distance. The fire judgment takes multiple source information fusion algorithm and infrared image recognition to improve adaptive of system on environment.

\section{REFERENCES}

[1] Fan-liu Yi, Shan-zhou Zun, "Tendency of Occurring of Ship-Fire and Its Reply Measure," World Shipping, vol. 24, no. 1, 2001, pp. 24-25

[2] Susan L., Rose Pehrsson R., Sean J., "Early warning fire detection system us-ing a probabilistic neural network," Fire Technology, vol. 39, 2003, pp. 147-171.

[3] X. Jiang, "Feature extraction for image recognition and computer vision," Proceedings of 2009 2nd IEEE International Conference on Computer Science and Information Technology, 2009, pp. 1-15.

[4] Cavallaro, A., "Image analysis and computer vision for undergraduates," Proceedings of IEEE International Conference on Acoustics, Speech, and Signal Processing, 2005, pp. 577-580.

[5] Jian-hua Zhao, Jun Fang, Xue-ming Shu, "An Identification Method of Fire Smoke Based on Neural Network," Acta Optica Sinica, vol. 23, no. 9, 2003, pp. 1086-1089.

[6] Yang Zhang, Hui Zhang, "Intelligent Fire Alarm System Based on Fuzzy Neural Net-work," Electronics Quality, vol. 4, 2004, pp. 4-5.

[7] Guan-lin Fan, "Design of fire alarm system based on multi-source information fusion," Proceedings of 3rd International Conference on Advanced Computer Theory and Engineering, 2010, pp. 269272.

[8] Naiwei Cheng, Qifeng Wu, "A Decision-Making Method for Fire Detection Data Fusion Based on Bayesian Approach," Proceedings of 2013 Fourth International Conference on Digital Manufacturing and Automation (ICDMA), 2013, pp. 21-23.

[9] James A. Milke, Matthew E., "Investigation of multi-sensor algorithms for fire detection," Fire Technology, vol. 39, 2003, pp. 363-382.

[10] Feng Sang, Guo-ping Jiang, Fang Wang, "Compound Intelligent Fire Detector Con-structed By Using BP Network," Process Automation Instrumentation, vol. 3, 2003, pp. 39-41. 